\title{
A General Methodology to Predict the Linear Rheology of Branched Polymers
}

\author{
E. van Ruymbeke, ${ }^{*}, \dagger$ C. Bailly, ${ }^{\ddagger}$ R. Keunings, ${ }^{\S}$ and D. Vlassopoulos ${ }^{\dagger, \|}$ \\ FORTH, Institute of Electronic Structure \& Laser, Heraklion, Crete, Greece, Unite de Physique et \\ Chimie des Hauts Polymères, Université catholique de Louvain, Louvain-la-Neuve, Belgium, CESAME, \\ Université catholique de Louvain, Louvain-la-Neuve, Belgium, and Department of Materials Science \& \\ Technology, University of Crete, Heraklion, Crete, Greece
}

Received February 28, 2006; Revised Manuscript Received July 7, 2006

\begin{abstract}
We present a general coarse-grained model for predicting the linear viscoelasic properties of branched polymers from the knowledge of their molecular structure and three viscoelastic parameters, i.e., the Rouse time of an entanglement segment, the plateau modulus, and the entanglement molecular weight. The model uses the ingredients of the tube-based theories of McLeish and co-workers, and its implementation is based on a timemarching algorithm; this conceptual approach was already successfully applied to linear and star polymers, and it is appropriately modified here to account for more complex branched architectures, within the framework of dynamic tube dilation (using the criteria of Graessley). Whereas the molecular physics behind this model is the well-established hierarchical tube-based motion, the new element is a different macromolecular coordinate system and account of the branch points diffusion. With proper account of polydispersity, successful description of a wide range of rheological data of $\mathrm{H}$ and pompom polymers is obtained, with the use of the dilution exponent $\alpha$ $=1$ and the parameter $p^{2}=1$. The proposed methodology thus represents a generic approach for predicting the linear rheology of branched polymers.
\end{abstract}

\section{Introduction}

Branched polymer rheology represents a very active field of research with important challenges, both from the scientific and technological viewpoints. Recent progress in this field was triggered by the advances in the tube-model theories ${ }^{1-7}$ and the availability of well-defined model polymers. ${ }^{8-12}$ It is now known that branched polymers relax hierarchically..$^{13-18}$ In other words, entanglements belonging to topologically different parts of the macromolecule (branches or backbone or different layers) relax in a certain sequence, obeying seniority rules according to which the outermost parts of the molecule (those with free dangling ends) relax first and the innermost ones last; the stress is fully relaxed only after the slowest segments have lost the memory of their initial orientation. For example, for the case of an H-polymers, ${ }^{14,19}$ the simplest hierarchical branched structure with a linear connector chain linking two grafted linear chains, the relaxation sequence is as follows: the end-grafted chains relax first (via starlike relaxation, as described by the MilnerMcLeish model ${ }^{20,21}$ ) while the backbone remains frozen, and eventually the backbone relaxes by reptation. The analysis is carried out in the context of dynamic tube dilation (DTD), ${ }^{20-23}$ according to which the relaxed branches act as solvent for the yet-unrelaxed backbone. However, the relaxed branches represent an extra friction for the branch point; the effect on the overall relaxation process is accounted for by the branch point diffusion, which has to precede the backbone reptation. The incorporation of the branch point diffusivity includes an unknown parameter, $p^{2} .^{24-26}$

Recently, a time-marching algorithm was developed to predict the linear viscoelastic properties of mixtures of linear and

\footnotetext{
* Corresponding author. E-mail: evelynvr@iesl.forth.gr.

FORTH, Institute of Electronic Structure \& Laser.

$\doteqdot$ Unite de Physique et Chimie des Hauts Polymères, Université catholique

$\S$ CESAME, Université catholique de Louvain.

"Department of Materials Science \& Technology, University of Crete.
} de Louvain. star polymers, as well as asymmetric star polymers. ${ }^{27}$ It is based on the tube model predictions of the fluctuations and reptation processes, which proceed simultaneously, but with different probabilities, without any imposed time scale separation; the solvent effect due to relaxed segments is treated as a constraint release effect based on the approach of Graessley. ${ }^{28-30}$ The model provides excellent predictions of the linear rheology for a wide range of linear polymer mixtures and asymmetric stars. ${ }^{27}$ More importantly, in the latter case there is no need to invoke the $p^{2}$ parameter (as there is no artificially imposed time scale separation), thus avoiding the related controversy. ${ }^{24-26,31,32}$

On the basis of this recent success, and keeping in mind the ongoing concerns about the value of the $p^{2}$ parameter for different branched polymers, ${ }^{24-26,31,32}$ as well as on the availability of accurate data sets with a variety of model branched polymers, we decided to expand this time-marching methodology to architecturally complex macromolecules. This is the scope of the present contribution, which focuses on the prediction of the linear rheology of pompom and (their special case) H-polymers, and compares with existing relevant experimental data.

The paper is organized as follows: After this introduction, we present in section II the main ingredients of the theoretical modeling, including the macromolecular coordinates, relaxation modes, dilution and polydispersity effects. Then, in section III we present and discuss the predictions of the model, and in particular we compare them against published experimental data for model $\mathrm{H}$ and pompom polymers. Finally, the main conclusions and consequences from this work are summarized in section IV.

\section{Theoretical Modeling}

II.1. Equilibrium State and Equilibrium Lengths. As already mentioned, he treatment of the friction around a branch point, due to the relaxed branches, is very important for the calculation of the fluctuations times of the backbone segments. 
Likewise, defining the effective backbone equilibrium length (after the relaxation of the branches), about which the real length of the backbone fluctuates, is also of great significance for the proper analysis of branched polymer relaxation. As already mentioned, DTD suggests that the polymer fraction already relaxed will act as a solvent for the relaxation of the remaining oriented part of the polymer. Therefore, the equilibrium state of the polymer evolves in time as a function of the unrelaxed part of the polymer, $\Phi(t):^{20,21,23}$

$$
\begin{gathered}
M_{\mathrm{e}}(t)=\frac{M_{\mathrm{e}}(0)}{\Phi(t)^{\alpha}} \\
L_{\mathrm{eq}}(t)=L_{\mathrm{eq}}(0)(\Phi(t))^{\alpha / 2} \\
a(t)=\frac{a(0)}{(\Phi(t))^{\alpha / 2}}
\end{gathered}
$$

where $\alpha$ is the dilution exponent, $M_{\mathrm{e}}(t)$, the effective molecular weight between two entanglements, $a(t)$, the effective tube diameter and $L_{\mathrm{eq}}(t)$, the effective equilibrium length of a subchain at time $t$ if its length is equal to $L_{\mathrm{eq}}(0)$ at time $t=0$. According to these equations, after the branches of a pompom molecule have relaxed, their effective equilibrium length is reduced in exactly the same way as the effective equilibrium length of the backbone or of the inner part of the backbone. This is a consequence of the fact that both branches and backbone are assumed to "see" the same environment:

$$
\begin{gathered}
L_{\text {eq,branch }}(t)=L_{\text {eq,branch }}(0)(\Phi(t))^{\alpha / 2}=\left(\frac{M_{\text {branch }}}{M_{\mathrm{e}}(0)} a_{0}\right)(\Phi(t))^{\alpha / 2} \\
L_{\text {eq,inner backbone }}(t)=L_{\text {eq,inner backbone }}(0)(\Phi(t))^{\alpha / 2}= \\
\left(\frac{M_{\text {inner backbone }} / 2}{M_{\mathrm{e}}(0)} a_{0}\right)(\Phi(t))^{\alpha / 2}(5) \\
L_{\text {eq,total backbone }}(t)=L_{\text {eq,inner backbone }}(t)+L_{\text {branch }}(t)
\end{gathered}
$$

where $L_{\text {eq,branch }}$ and $L_{\text {eq,inner backbone }}$ are the equilibrium length of one branch and of the half-inner part of the backbone, and $\Phi(t)$ is the unrelaxed fraction of the polymer at time $t$.

According to these eqs, the equilibrium length of the branches just after their relaxation is nonzero $\left(\Phi\left(\tau_{\text {branch }}\right) \approx \Phi_{\text {inner backbone }}\right)$. Therefore, the equilibrium location of the real end of the backbone chain after the relaxation of the branches is not at the actual branching point. It is defined by the equilibrium length

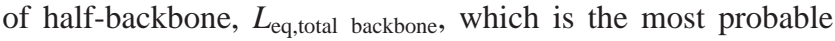
length of the half-molecule, not necessarily the shorter length that the half-molecule has taken (first passage problem). Another way to consider the situation is to say that, after the relaxation of the branches, the chain ends will not fluctuate around the branching points but around the relaxed branch ends instead.

In the conventional molecular coordinate system used in the literature, ${ }^{13-19}$ a backbone segment is defined by the normalized variable $x_{\mathrm{b} \text {,inner }}$ ranging from 0 at the branching point to 1 at the middle of the molecule. This means that, after the relaxation of the branches, the most stable configuration of a pompom molecule is obtained when the chain ends reach their branching point, about which they fluctuate. However, this definition contradicts that of the equilibrium length. A related problem is the fluctuations process of the backbone, where we need to consider that the fluctuations time of a molecular segment close to the branching point but inside the backbone (between the

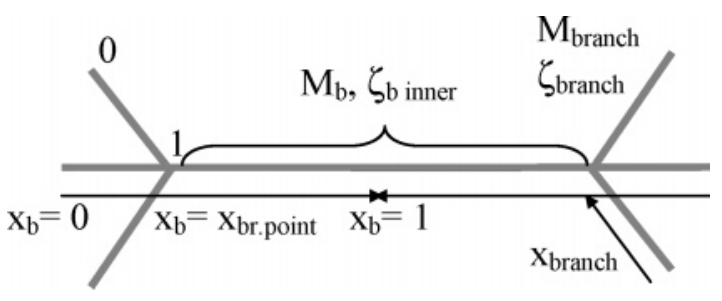

Figure 1. Macromolecular coordinates and definitions for a pompom molecule.

two branching points) will be shorter than the respective time of another segment equally close to the branching point but on the branch side (see Figure 1 below). Therefore, in the following, we will use another way to define a backbone segment.

II.2. Time Marching Algorithm and Relaxation Moduli. The polymers analyzed in this work have $\mathrm{H}$ or pompom architecture. As illustrated in Figure 1, they consist of a backbone and $q$ branches at each of their ends. The inner part of the backbone has a molecular weight of $M_{\mathrm{b}}$ and each branch has a molecular weight of $M_{\text {branch. }}$. We consider here only molecules with branches of the same length. Note that for $q=$ 2 we have an $\mathrm{H}$-polymer.

Each molecular segment is defined by the normalized variable $x$. For a branch segment, the variable $x_{\text {branch }}$ goes from 0 at the free end of the branch to 1 at the branching point. The choice of the starting point of the backbone is very important and can have a strong impact on the predictions (via the potential). Here, to be consistent with the definition of the equilibrium length of the backbone, we chose the branch free-end as the starting point for the definition of $x_{\mathrm{b}}=0$ (see Figure 1), and the middle of the molecule as the ending point, $x_{\mathrm{b}}=1$. Therefore, the (effective) backbone is defined as the longest way from an end to another end of the molecule, on which $2(q-1)$ branches are fixed (at distances $x_{\text {branch }}$ from the free ends), and their respective volumetric fractions, $\varphi_{\mathrm{b}}$ and $\varphi_{\text {branch }}$, are

$$
\begin{gathered}
\varphi_{\mathrm{b}}=\frac{M_{\mathrm{b}}+2 M_{\mathrm{branch}}}{M_{\mathrm{b}}+2 q M_{\mathrm{branch}}} \\
\varphi_{\text {branch }}=\frac{2(q-1) M_{\mathrm{branch}}}{M_{\mathrm{b}}+2 q M_{\mathrm{branch}}}
\end{gathered}
$$

The relaxation function $G(t)$ of the polymer is determined by using the time-marching algorithm, which sums up all contributions over all branches and positions along the branches. ${ }^{27}$ This is explained in some detail in Appendix I. In a similar way, to include the contribution of the (relaxed) polymer solvent to the reptation and fluctuations processes (see the next sections below), we determine the unrelaxed fraction of the polymer $\Phi\left(t_{k}\right)$ at each time step $t_{k}$ as

$$
\begin{array}{r}
\Phi\left(t_{k}\right)=\varphi_{\mathrm{b}} \int_{0}^{1}\left(p_{\text {rept }}\left(x_{\mathrm{b}}, t_{k}\right) p_{\text {fluc }}\left(x_{\mathrm{b}}, t_{k}\right)\right) \mathrm{d} x_{\mathrm{b}}+ \\
\varphi_{\text {branch }} \int_{0}^{1}\left(p_{\text {fluc }}\left(x_{\text {branch }}, t_{k}\right)\right) \mathrm{d} x_{\text {branch }}
\end{array}
$$

where $p\left(x_{i}, t_{k}\right)$ is the survival probability of a segment $x_{i}$ (by reptation or by fluctuations) at time $t_{k}$.

II.3. Tube Length Fluctuations and Branching Point Diffusion. Branch Fluctuations. The branch fluctuations for a pompom molecule follow exactly the same rules as the fluctuations of the arms of a star polymer, which has been already addressed. ${ }^{27}$ The particular details for the pompom case are presented in Appendix II. 
Backbone Fluctuations: The Two Fluctuations Modes. Segments along the (entire) backbone of a pompom molecule can relax according two different modes: one is with respect to the branching point, which involves the normal segmental friction, and one with respect to the middle of the molecule, which takes into account the extra friction due to the relaxing branches, and thus hinders the branching point motion.

The former mode describes fluctuations of the equilibrium length of the outer part of the backbone with respect to the branching point. It considers only segments $x_{b}$ localized beyond the branching point (in the branch region), i.e., those between 0 and $x_{\text {br.point }}$ (see Figure 1). These fluctuations are described exactly as those of a star arm and are calculated by using eqs II-3 to II-9 (see Appendix II), after rescaling of the variable $x_{\mathrm{b}}$ into $x_{\text {branch }}$, going from 0 at the branch end to 1 at the branching point:

$$
x_{\text {branch }}=\frac{x_{\mathrm{b}}\left(M_{\mathrm{branch}}+M_{\mathrm{b}} / 2\right)}{M_{\mathrm{branch}}}
$$

The latter mode describes fluctuations of the equilibrium length of the half molecule, i.e., from the end of the extended backbone to the middle of the molecule ( $x_{\mathrm{b}}$ ranging from 0 to 1 ). Indeed, as already explained before section II.1, even if the branches are relaxed, their equilibrium length is nonzero; it is therefore important to consider the tube length fluctuations of the entire backbone. This fluctuations mode requires the motion of the branching point, where the branches are covalently bonded, and which is able to move only at the time scale of the fluctuations time of the branches, $\tau_{\text {branch }}(1)$. This effect must be included in the total friction coefficient, $\zeta_{\text {tot }}$, felt by the backbone: ${ }^{24-26}$

$$
\xi_{\text {tot }}=(q-1) k T \frac{2 \tau_{\text {branch }}(1)}{a^{2}}+\zeta_{0}\left(\frac{M_{\mathrm{b}} / 2+M_{\text {branch }}}{m_{0}}\right)
$$

where $a$ is the length of a segment between two entanglements, $\zeta_{0}$ is the monomeric friction coefficient and $m_{0}$ is the monomeric molecular weight. The first term is the friction contribution arising from the branches and the second term the friction arising from the backbone itself. The latter contribution is usually negligible, unless the branches of the pompom molecules are very short branches in comparison to the backbone.

Because of this additional friction coming from the branches, the early fluctuation times of the backbone segments are slowed. ${ }^{17,26}$ This is taken into account by adding an "delay" time, $\tau_{\text {delay }}$, to the Rouse time of the chain in eq II-7:

$\tau_{\text {early }}\left(x_{\mathrm{b}}\right)=\frac{9 \pi^{3}}{16}\left(\frac{M_{\mathrm{b}} / 2+M_{\mathrm{branch}}}{M_{\mathrm{e}}}\right)^{2}\left(\tau_{\mathrm{R}, \text { chain }}+\tau_{\text {delay }}\right) x_{\mathrm{b}}{ }^{4}$

This delay time is easily determined from the fact that the branches take a time proportional to $(q-1) \tau_{\text {branch }}(1)$ to retract and that each retraction allows the chain end to cover a distance equal to $a$, the distance between two entanglements. Since to be completely relaxed by the Rouse process, the chain end has to cover a distance equal to $L_{\mathrm{eq}}(=a Z)$, we have

$$
\begin{aligned}
\tau_{\text {delay }}=\frac{2}{3 \pi^{2}}(q-1) \tau_{\text {branch }}(1) Z= \\
\\
\qquad \frac{2}{3 \pi^{2}}(q-1) \tau_{\text {branch }}(1)\left(\frac{M_{\mathrm{b}} / 2+M_{\text {branch }}}{M_{\mathrm{e}}}\right)
\end{aligned}
$$

From eqs 12 and 13, we obtain

$$
\begin{aligned}
& \tau_{\text {early }}\left(x_{\mathrm{b}}\right)=\frac{3 \pi(q-1)}{8}\left(\frac{M_{\mathrm{b}} / 2+M_{\text {branch }}}{M_{\mathrm{e}}}\right)^{3} \tau_{\text {branch }}(1) x_{\mathrm{b}}{ }^{4}+ \\
& \frac{9 \pi^{3\left(M_{\mathrm{b}} / 2+M_{\text {branch }}\right)^{4}}}{16} K_{\mathrm{e}}^{2} K_{\text {Rouse }} x_{\mathrm{b}}{ }^{4}
\end{aligned}
$$

On the other hand, the activated (late) fluctuations times of the backbone are determined in the same way as for the branches (see Appendix II)

$$
\begin{array}{r}
\frac{\partial \ln \tau_{\text {late }}\left(x_{\mathrm{b}}\right)}{\partial x_{\mathrm{b}}}=3\left(\frac{M_{\mathrm{b}} / 2+M_{\text {branch }}}{M_{\mathrm{e}}(t)}\right) x_{\mathrm{b}}= \\
3\left(\frac{M_{\mathrm{b}} / 2+M_{\text {branch }}}{M_{\mathrm{e} 0}}\right) x_{\mathrm{b}} \Phi\left(t_{k}, x_{\mathrm{b}}\right)^{\alpha}
\end{array}
$$

where the unrelaxed part of the polymer $\Phi\left(t_{k}, x_{\mathrm{b}}\right)$, which does not act as a solvent for the relaxation of segment $x_{\mathrm{b}}$ is ${ }^{5,21}$

$$
\begin{aligned}
& \Phi\left(t_{k}, x_{\mathrm{b}}\right)=\varphi b\left(1-x_{\mathrm{b}}\right)+ \\
& \varphi_{\text {branch }} \max \left(0,1-\sqrt{\frac{M_{\mathrm{b}} / 2+M_{\text {branch }}}{M_{\text {branch }}}} x_{\mathrm{b}}\right)
\end{aligned}
$$

Because the reptation of the backbone takes place only at the end of the pompom polymer overall relaxation, we can neglect its effect on the fluctuations times and $\Phi\left(t_{k}, x_{\mathrm{b}}\right)$ reflects only the fluctuations process. The transition between the two fluctuation processes occurs at a transition segment for which the potential is equal to $k T$, and is described by eqs II- 8 and II-9 in Appendix II.

Backbone Fluctuations: From the First to the Second Mode. While, in principle, segments localized beyond the branching point can move according to the two fluctuations modes, the second one (with respect to the chain middle point) is so slow in comparison to the first one (with respect to the branch point) that we can neglect it: backbone segments localized beyond the branching point (outer part of backbone, branches region) will relax via the tube length fluctuations of the outer part of the molecule (branches), with respect to the branching point. On the other hand, segments localized on the inner part of the backbone will relax according to the second fluctuations process with respect to the middle point. However, the transition between the two modes leads to a discontinuity of the segments fluctuations times at the branching point, the second mode assuming a value of $\tau_{\text {backbone }}\left(x_{\text {br. point }}\right)>\tau_{\text {branch }}(1)$ (see Figure 3 below). To account for the fact that a chain end will diffuse faster to its branching point than expected from the second fluctuations mode only, we defined an "equivalent pompom molecule" with shorter branch length, such as the second fluctuations mode predicts that the chain ends reach the branching point after a time equal to $\tau_{\text {branch }}(1)$. This is schematically illustrated in Figure 2: the length of the equivalent branches $\mathrm{M}_{\mathrm{i}}$ is fixed so that the chain ends of the "equivalent pompom molecule" take a time equal to $\tau_{\text {branch }}(1)$ to diffuse up to the branching point, while feeling the total friction $\zeta_{\text {tot }}$. Therefore, to calculate the fluctuations times of the segments localized on the inner part of the backbone, i.e., the segments from $x_{\mathrm{b}}=x_{\text {br.point }}$ to $x_{\mathrm{b}}=1$, the backbone segments are described

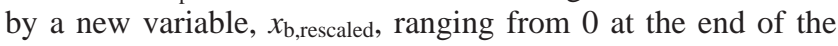
branches of the equivalent molecule to 1 at the middle of the backbone. Therefore, in eqs 14 and 15, the molecular weight 


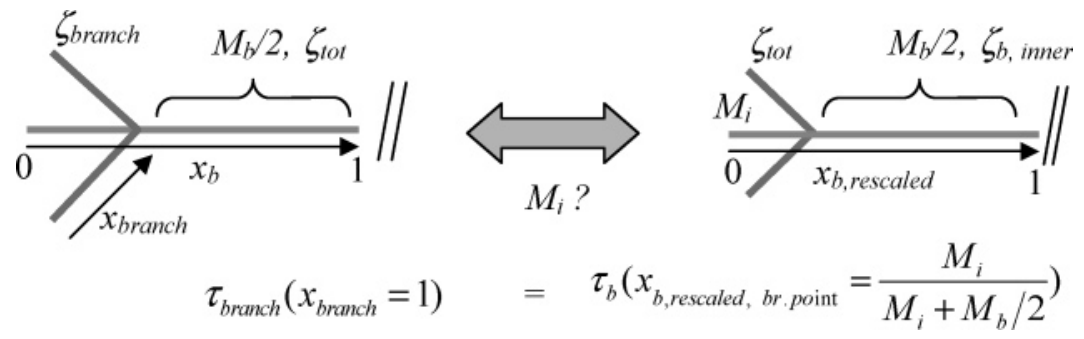

Figure 2. Definition of an "equivalent pompom molecule" used to calculate the fluctuations times of the segments localized in the inner part of the backbone.

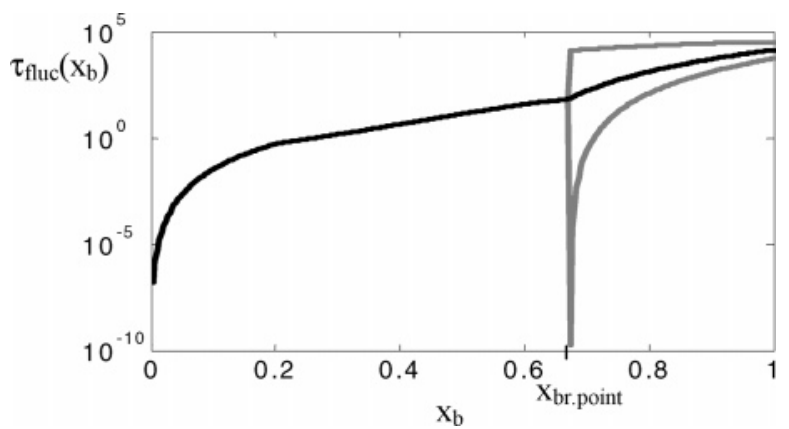

Figure 3. Fluctuation times of the segments along the extended backbone, by considering $x_{\mathrm{b}}=0$ at the branching point (-- ), or by considering $x_{\mathrm{b}}=0$ at the end of a branch, with $(-)$ or without $(\cdots)$ rescaling $x_{\mathrm{b}}$ to calculate the fluctuations times of the inner part of the backbone.

of a branch, $M_{\text {branch }}$, must be replaced by $M_{i}$ and the reference variable $x_{\mathrm{b}}$, by the variable $x_{\mathrm{b}, \text { rescaled: }}$

$$
x_{\mathrm{b}, \text { rescaled }}=\frac{x_{\mathrm{b}}\left(M_{\mathrm{branch}}+M_{\mathrm{b}} / 2\right)-M_{\mathrm{branch}}+M_{i}}{\left(M_{i}+M_{\mathrm{b}} / 2\right)}
$$

We now obtain a continuous curve to describe the fluctuations times of the segments along the entire backbone, as shown in Figure 3.

II.4. Reptation of the Backbone. The probability that a segment of the backbone relaxes by reptation during a time interval $\Delta t$ has been described by Doi and Edwards. ${ }^{2}$ It is a function of the reptation time $\tau_{\text {rept }}$ of the molecule:

$$
p\left(x_{i}, t\right)=\sum_{n \text { odd }} \frac{4}{p \pi} \sin \left(\frac{n \pi x_{i}}{2}\right) \exp \left(\frac{-n^{2} \Delta t}{\tau_{\text {rept }}}\right)
$$

For a pompom molecule, the reptation time of the backbone is determined by taking into account the friction of the backbone itself as well as that due to the relaxed branches, in a way comparable to that used for asymmetric star molecules ${ }^{27}$

$$
\begin{aligned}
& \tau_{\text {rept }, 0}= \\
& \quad \frac{L_{\text {eq }, 0}^{2}}{\pi^{2}}\left(\frac{1}{D_{\text {backbone }}}+\frac{2(q-1)}{D_{\text {branch }}}\right)=3 \tau_{\mathrm{e}}\left(\frac{M_{\mathrm{b}}+2 M_{\text {branch }}}{M_{\mathrm{e}, 0}}\right)^{3}+ \\
& \frac{2(q-1) \tau_{\text {branch }}(1)}{\pi^{2}}\left(\frac{M_{\mathrm{b}}+2 M_{\text {branch }}}{M_{\mathrm{e}, 0}}\right)^{2}=\tau_{\text {rept }, 0, \mathrm{~b}}+\tau_{\text {rept }, 0, \mathrm{a}}
\end{aligned}
$$

where $\tau_{\mathrm{e}}$ is the Rouse time of an entanglement segment. As explained in ref 27 , we consider that a chain is relaxed by reptation when its center of mass has diffused a distance $L_{\text {eq. }}$. along the tube, while most papers consider that the diffusion of the center of mass occur on a distance $L_{\mathrm{eq}}\left(1-x_{\mathrm{d}}\right)$, where $x_{\mathrm{d}}$ is the fractional distance of the deepest segment relaxed by fluctuations at that time. The fact that contour length fluctu- ations speed up the total relaxation process of the polymer is rather taken into account in separated term (see eq I.2) than in the expression of the reptation time (eq 19). This way of including contour length fluctuations and reptation in two separated terms is based on the fact that on the average, the center of mass of a molecule does not change its position, and that the primitive path of each molecule has an average length equal to its equilibrium length $L_{\text {eq,molecule. Some segments can }}$ be relaxed by both reptation and fluctuations. However, these two relaxation mechanisms are not necessarily additive: if fluctuations occur toward deeper segments (that means that the real length of the molecule is smaller than $L_{\mathrm{eq} \text {,molecule }}$ ) during the reptation of the molecule can accelerate the reptation process (see Figure 4, left); on the other hand, fluctuations toward the other direction (that means that the real length of the molecule becomes larger than $L_{\text {eq,molecule}}$ ), which happens with the same probability, will have the opposite effect (see Figure 4, right). Since fluctuations far from the equilibrium length (for example, fluctuations of the segment $x_{\mathrm{d}}\left(\operatorname{such}\right.$ as $\left.\tau_{\text {fluc }}\left(x_{\mathrm{d}}\right)=\tau_{\text {rept }}\right)$ ) cannot be considered fast compared to the reptation time, we cannot assume that the chain will fluctuate again toward a real length of $L_{\text {eq.,molecule }}\left(1-x_{\mathrm{d}}\right)$ during the reptation process. Therefore, it seems more reasonable to consider that the molecule reptates along its equilibrium length and not along the shorter length that it has taken at time $t=\tau_{\text {rept }}$ (which is a first passage problem).

When there is a large time scale separation between the two different relaxation processes, the backbone feels the relaxed branch fraction acting as a solvent during its reptation: ${ }^{13}$

$$
\tau_{\text {rept }}(t)=\tau_{\text {rept }, 0, \mathrm{~b}} \Phi_{\text {active }}(t)+\tau_{\text {rept }, 0, \text { branch }}\left(\Phi_{\text {active }}(t)\right)^{2}
$$

The value of $\Phi_{\text {active }}(t)$ stems from the extended criteria of Graessley, ${ }^{27,28}$ according to which a relaxed polymer fraction $\Phi(t)$ will act as a solvent for the remaining part of the (unrelaxed) polymer only if their relaxation processes are well separated in time. Indeed, the tube can be dilated by the slow removal of entanglements, but reptation within the dilated tube requires correlated release of entanglement constraints and can only occur if constraint release is fast with respect to the reptation time of the test chain. This condition is verified if the reptation time of the backbone is larger than its constraint release-Rouse time associated with its partially relaxed state corresponding to an unrelaxed proportion $\Phi(t)$; the ratio of the two times is called the Graessley number ${ }^{27,31-33}$ and is given by

$$
G r=\frac{\tau_{\text {rept }}(t)}{\tau_{\text {CRRouse,backbone }}(\Phi(t))}=\frac{\tau_{\text {rept }}(t)}{t_{\Phi} Z_{\mathrm{b}}^{2}}>1
$$

where $Z_{\mathrm{b}}$ is the number of backbone segments and $t_{\Phi}$ is the necessary time for a segment to reach a probability of being relaxed by constraint release equal to $(1-\Phi(t))$ (thus, $t_{\Phi}=t$ ). 


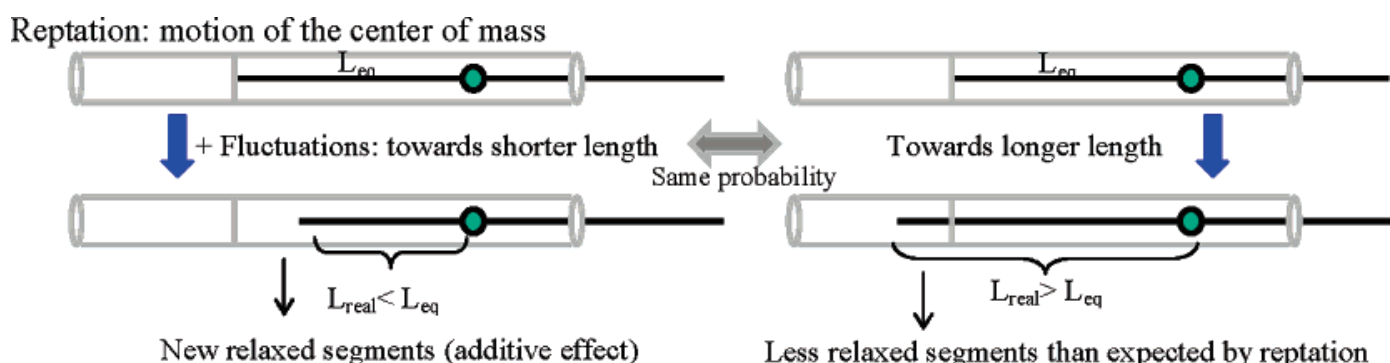

Figure 4. Interaction between reptation and the contour length fluctuations; left: additive effect, right: nonadditive effect.

Alternatively, at time $t$, the polymer fraction, $\Phi_{\text {active }}(t)$, which acts as a solvent in the reptation process of the backbone, is equal to the relaxed polymer fraction $\Phi\left(t / Z_{\mathrm{bac}}{ }^{2}\right.$ ) (see eq 9). Because this later value evolves through time, the reptation time of the backbone is not constant, and this is taken into account by the time-marching algorithm. ${ }^{27}$

Consequently, $\Phi_{\text {active }}(t)$ in eq 20 is equal to the unrelaxed polymer fraction at time $t /\left(\left(M_{\mathrm{b}}+2 M_{\text {branch }}\right) / M_{\mathrm{e}}\right)^{2}$. As will be shown in section III below, this criterion influences the predictions of the relaxation moduli a great deal. As already discussed in the context of linear polymer mixtures, ${ }^{27}$ the reptation time considered here is not proportional to $\left(1-x_{\text {rel }}(t)\right)$, where $x_{\text {rel }}(t)$ is the deeper inner backbone segment which is relaxed by fluctuations at time $t$, but instead it is considered along the equilibrium length of the backbone.

II.5. Constraint Release and Dynamic Tube Dilation. The constraint release effect is known to play an important role in the relaxation of the polymer. ${ }^{20-23}$ Its influence can be accounted for in two different ways: The first, which is a global effect, is based on the fact that each entanglement is formed by two segments and that the relaxation of one of these disentangles the other one, enabling its "early" relaxation. ${ }^{22,23,29,30}$ This constraint release effect can be considered as "global" effect, as we can assume that each segment senses the same environment and thus, has the same probability of relaxing in this way. This implies an increase of the effective molecular weight between two such entanglements (which have an effective role in the orientation of the molecule), a decrease of the equilibrium length of the molecules and a increase of the tube diameter (see eqs $1-3)$. This is in fact the dynamic tube dilution, introduced by Marrucci $^{22}$ and developed by Ball and McLeish. ${ }^{23}$ In this work, this important effect is taken into account in the term $p_{\text {envir. }}\left(x_{i}, t\right)$ of eq I.2. ${ }^{27}$ This term is nearly equal to the unrelaxed fraction of the polymer (see eq 9), and represents the conditional probability that any random segment in the polymer is still oriented, if we know that the segment $x_{i}$ (see eq I-2) is not relaxed (either by reptation or by fluctuations):

$$
\begin{gathered}
p(x, t)=\left[\varphi _ { \mathrm { b } } \left(\int_{0}^{x_{\text {equiv }, \mathrm{b}}\left(x_{\mathrm{b}}\right)}\left(p_{\text {rept }}\left(x_{\mathrm{b}}, t\right) p_{\text {fluc }}\left(x_{\mathrm{b}}, t\right)\right) \mathrm{d} x_{\mathrm{b}}+\right.\right. \\
\left.\int_{x_{\text {equiv } \mathrm{b}}\left(x_{\mathrm{b}}\right)}^{1} p_{\text {rept }}\left(x_{\mathrm{b}}, t\right) \mathrm{d} x_{\mathrm{b}}\right)+ \\
\varphi_{\text {branch }}\left(\int_{0}^{x_{\text {equi branch }}\left(x_{\text {branch }}\right)} p_{\text {fluc }}\left(x_{\text {branch }}, t\right) \mathrm{d} x_{\text {branch }}+\right. \\
\left.\left.\int_{x_{\text {equiv branch }}\left(x_{\text {branch }}\right)}^{1} 1 \mathrm{~d} x_{\text {branch }}\right)\right]
\end{gathered}
$$

The segments $x_{\text {equiv,b. }}\left(x_{i}\right)$ and $x_{\text {equiv,branch }}\left(x_{i}\right)$ are potentially equivalent to the probe segment $x_{i}$. If the branch is completely relaxed, then $x_{\text {equiv,branch }}\left(x_{i}\right)=1$.

In addition, the constraint release mechanism influences the reptation and fluctuations times of the molecules, as already described by the parameter $\Phi$ above. However, the disoriented (relaxed) part of the polymer cannot be immediately considered as a solvent for these relaxation mechanisms: the "fast" segments act effectively as a solvent for the "slow" segments only if the latter have had enough time to explore all the conformations in their dilated tube. This means that occupying this dilated tube without being able to move freely inside is not a sufficient condition to consider the (accelerated) relaxation of the "slow" segments within the dilated tube. This idea, introduced by Graessley ${ }^{28}$ for mixture of two linear chains and confirmed by McLeish, ${ }^{23}$ Watanabe, ${ }^{31,32}$ and Park and Larson ${ }^{33}$ also works well for branched polymers. ${ }^{27}$ Therefore, in this work, we consider $\Phi_{\text {active }}$ instead of $\Phi$ to determine the reptation time (see eq 20), in order to conform with this criterion. Since the initial fluctuations times of the segments are exponentially separated, we assume, in this work, that they are not affected by this criterion. In the case of a pompom or $\mathrm{H}$ molecule considered here, only the deepest segments, near the middle of the molecule, can be sensitive to this criterion. However, since this part of the molecule relaxes by reptation, this does not affect the predictions. In general, however, for other architectures such as treelike polymers, this criterion must be accounted for.

II.6. Polydispersity. The pompom and H-polymer samples analyzed in the next section are assumed to be monodisperse. This is based on the characterization ${ }^{34-36}$ of these samples and the widely used hypothesis that samples with $M_{\mathrm{w}} / M_{\mathrm{n}}<1.1$ can be treated as truly monodisperse ones. ${ }^{7,13,27}$ Nevertheless, it has been shown that in branched polymers, in particular, this is not necessarily true, and polydispersities as low as 1.05 can influence the rheological predictions significantly. ${ }^{17,18}$ In this work, because of the latter, as well as our own observations of the high sensitivity of the fluctuations process to the molecular weight, we chose to include this effect in the model by considering a constant polydispersity of 1.05 in both the branches and the backbone for each sample.

We used a Wesslau distribution ${ }^{37}$ for determining the molecular weight of the branches and of the inner part of the backbone (between the two branching points). As shown in Figure 5, we considered five molecular weights in a distribution, each of them representative of one-fifth of the overall distribution. Assuming that all branches of the same molecule have the same molecular weight, we thus worked with 25 different molecules in same proportion in the polymer. Next, we used the eqs described above to determine the relaxation times of each kind of molecules, with only a few modifications:

(i) The variable $\Phi(x, t)$ was determined for all the molecules. To define the part of each branch relaxed by fluctuations, we used the potential equivalence between the molecules, introduced in part II.3. For simplicity in the calculations of $\Phi(t)$, we assumed that none of the backbone segments relaxes before the relaxation of the branches in all the molecules is completed. Since the polydispersity is very small, this approximation did not affect the results. 


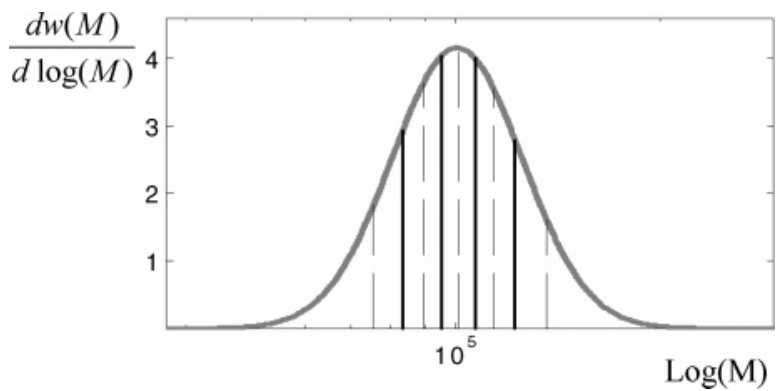

Figure 5. Normalized Wesslau molecular weight distribution. The overall distribution is divided to five equivalent surfaces (separated by the black continuous lines). Each of the surfaces is represented by its average molecular weight (- - ).

(ii) Equations 9, 16, 22, I-2, I-6, and II-5 were extended to consider every molecule.

Figure 6 depicts the effects of the polydispersity, first on the backbone, next on the branches, then on both the backbone and the branches. One can observe that polydispersity smears out the relaxation peak, in the sense that it makes it weaker and much broader. Moreover, its effect is larger on the branches than on the backbone. This is due to the large influence of the branch molecular weight on the motion of the branching point. Also, at high frequencies, where local motions are probed, there are no effects of polydispersity.

\section{Results and Discussion}

III.1. Parameters and Predictions. H-Polystyrene Melts. To assess the model in more detail, we compared predictions with available experimental relaxation moduli from different sets of polymers ( $\mathrm{H}$ or pompom). The first set is composed of several polystyrene $\mathrm{H}$-polymers synthesized and measured by Roovers. ${ }^{34}$ Their average molecular weights are listed in Table 1 (samples PS1-PS5). ${ }^{34-36,38}$

Backbone and branches of these samples have the same molecular weight. The model has two parameters that need to be defined: the entanglement molecular weight, $M_{\mathrm{e}}$, and the Rouse time of a segment $\tau_{\mathrm{e}}$. We used consistently $\tau_{\mathrm{e}}=8 \times$ $10^{-4} \mathrm{~s}, M_{\mathrm{e}}=16000 \mathrm{~g} / \mathrm{mol}$ (at $T_{\text {ref }}=169.5^{\circ} \mathrm{C}$ ). The value of the plateau modulus, $G_{N}{ }^{0}$, was $2.3 \times 10^{5} \mathrm{~Pa}$. Given that the density of the PS is $0.959 \mathrm{~kg} / \mathrm{m}^{3}$, this value is consistent with $^{7,17,39,40} G_{N}{ }^{0}=(4 / 5)\left(\rho R T / M_{\mathrm{e}}\right)$ within $15 \%$. Such a small deviation is acceptable and in line with what has been reported in the literature when using similar modeling efforts to predict polymer rheology. ${ }^{41}$ The origin of this small deviation remains a puzzle at present, but two issues that should be considered are the small experimental uncertainties in the high-frequency rheometrical measurements and the fact that the coupling between the faster Rouse relaxation of an entanglement segment and the branches relaxation (which can start before the former relaxes fully) are not taken into account. Furthermore, we fixed the value of the dilution exponent $\alpha$ to $1 .{ }^{42} \mathrm{We}$ decided to keep $\alpha$ constant for all the polymers analyzed in this work in order to test the predictive capability of the model without having an additional parameter. Since there is no real consensus on the value of this exponent, we fixed its value to 1 as proposed in refs $13,14,17,18$, and 24 . Results obtained with this set of parameters are shown in Figure 7. Very good agreement between experimental data and the model predictions was found for all samples without any adjustable parameters. This is already a departure from the earlier approaches which used a sensitive adjustable parameter, $p^{2}$. To ensure the universal validity of the model, we also tested two linear PS samples, again synthesized and measured by Roovers and co-workers, ${ }^{34,38}$ at the same
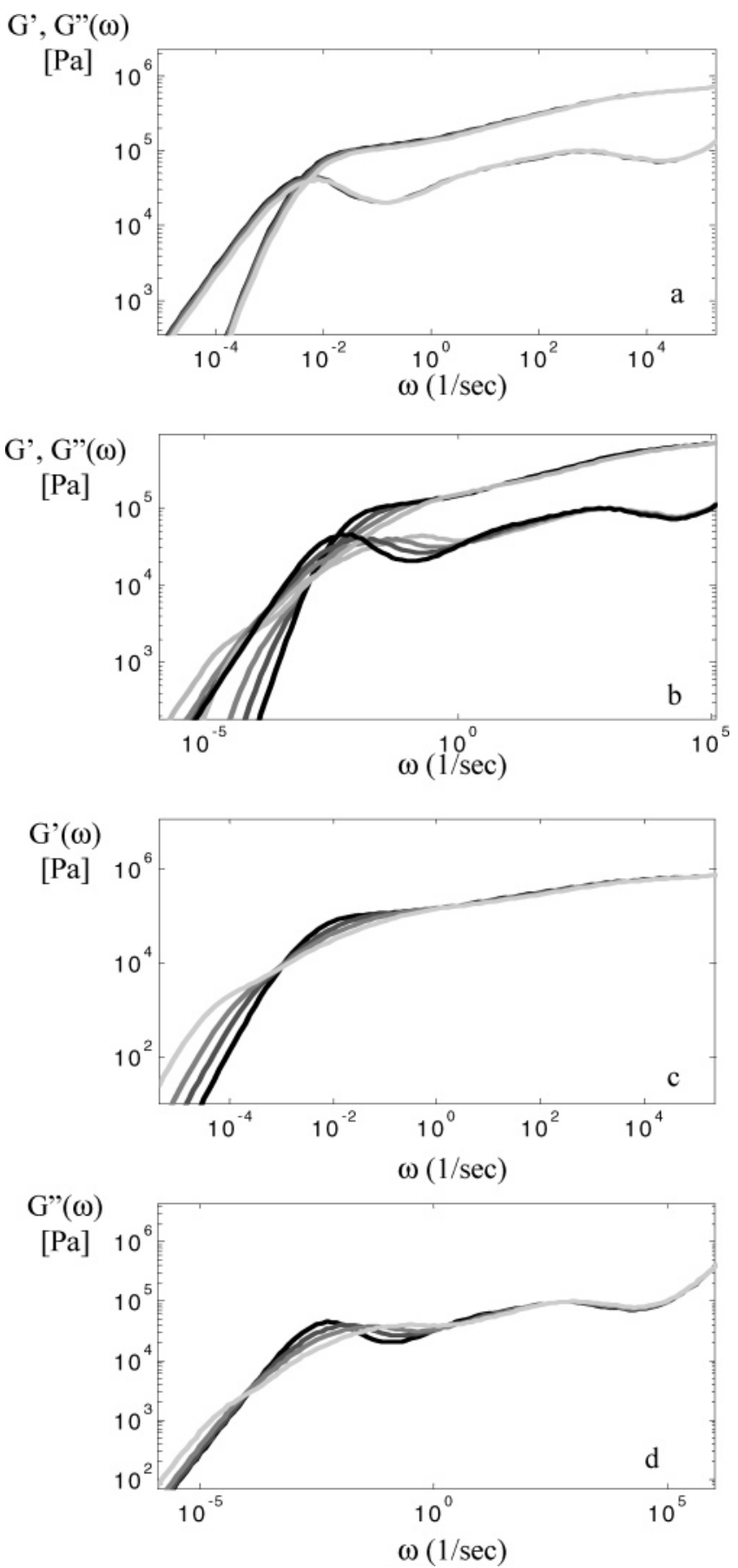

Figure 6. Model predictions of the relaxation moduli of samples PB4 (see Table 1) for different values of polydispersity: $1,1.05,1.1$, and 1.2 (from black to light gray). Polydispersity is considered (a) for the backbone molecular weight only; (b) for the branch molecular weight only; (c and d) for both the backbone and the branch molecular weights.

temperature (see Table 1, samples PS6 and PS7). Comparison between model predictions and experimental data are shown in Figure $7 d$, with the same set of parameters. Note that a linear polymer is a limiting case of this model, i.e., an H-molecule with branches of zero length.

Pompom Polybutadiene Melts and Solutions. The particular molecules have three branches on each side of the backbone ( $q$ $=3$ ) and are described in Table 1 (see samples PB1-PB4). ${ }^{35}$ The rheological properties were measured at $24.5^{\circ} \mathrm{C} .{ }^{33}$ Because they contain a large fraction of 1,2-microstructure (about 50\%), the value of their plateau modulus $\left(G_{N}{ }^{0}=700000 \mathrm{~Pa}\right)$ was found low compared to the typical value of $1 \mathrm{MPa}$ for 1,4- 
Table 1. Molecular Characteristics of the Samples Used

\begin{tabular}{crclcc}
\hline sample & $M_{\mathrm{b}}(\mathrm{g} / \mathrm{mol})$ & $M_{\text {branch }}(\mathrm{g} / \mathrm{mol})$ & $q$ & $T_{\text {ref }}\left({ }^{\circ} \mathrm{C}\right)$ & ref \\
\hline PS1 & 19000 & 19000 & 2 & 169.5 & 34 \\
PS2 & 44000 & 46000 & 2 & 169.5 & 34 \\
PS3 & 100000 & 103000 & 2 & 169.5 & 34 \\
PS4 & 123000 & 132000 & 2 & 169.5 & 34 \\
PS5 & 204000 & 205000 & 2 & 169.5 & 34 \\
PS6 & 275000 & & linear & 169.5 & 34 \\
PS7 & 800000 & & linear & 169.5 & 38 \\
PB1 & 47000 & 14800 & 3 & 24.5 & 35 \\
PB2 & 47000 & 19500 & 3 & 24.5 & 35 \\
PB3 & 90700 & 12000 & 3 & 24.5 & 35 \\
PB4 & 90700 & 19500 & 3 & 24.5 & 35 \\
PB5 & 86000 & & linear & 24.5 & 35 \\
PB6 & 129000 & & linear & 24.5 & 35 \\
PB7 & 176000 & & linear & 24.5 & 35 \\
PB8 & 89000 & 21000 & 3 & 23 & 36
\end{tabular}

$\mathrm{Pbd} ;{ }^{17,39,40}$ the corresponding value of $M_{\mathrm{e}}$ is higher and amount to $2250 \mathrm{~g} / \mathrm{mol}$. We used $\alpha=1$ and $\tau_{\mathrm{e}}=9 \times 10^{-7} \mathrm{~s}$ at $T_{\text {ref }}=$ $24.5{ }^{\circ} \mathrm{C}$. Results are shown in Figure 8. It is evident that predictions and experiments are in very good agreement.

Again, to test the universality of the model, the linear rheology of three linear polybutadienes from the same source (Table 1, samples PB5-PB7 $)^{35}$ was successfully predicted using the same set of parameters. The results are shown in Figure 9.

The last set of data presented in this work concerns the linear viscoelasticity of a pompom polybutadiene diluted in an oligomeric solvent (a 1,4-polybutadiene with $M_{\mathrm{w}}=1000 \mathrm{~g} / \mathrm{mol}$ ) at different concentrations, as described in Table 1 (sample PB8). Parameters used here are $M_{\mathrm{e}}=2050 \mathrm{~g} / \mathrm{mol}$ and $\tau_{\mathrm{e}}=3 \times 10^{-7} \mathrm{~s}$ at $T_{\text {ref }}=23{ }^{\circ} \mathrm{C}$. From eq 22 , the plateau modulus is $0.83 \mathrm{MPa}$. In this case, because a solvent is used, the (static) dilution concept works well. Very good agreement between experience and theory was found.

III.2. Influence of the Reptation on the Backbone Relaxation: Failure of Dynamic Dilution? From a closer inspection of the experimental data for the $\mathrm{H}$ and the pompom molecules (Figures 7, 8, and 10) around the terminal region, which is expected to be governed by the reptation process, it appears that the loss modulus exhibits a very broad maximum and that the storage modulus also exhibits a broad low-frequency relaxation. This behavior is reminiscent of relaxation by fluctuations (e.g., in star polymers), ${ }^{5}$ suggesting that there is virtually no reptation contribution to the terminal flow (or too small contribution, if any). This is tested below:

Figure 11 shows the comparison of the measured viscoelastic moduli of sample PS3 (see Table 1) with the respective predictions in three different cases: first, by taking into account the reptation process as described as in section II.4; next, by assuming that the backbone of the pompom molecule relaxes by reptation in its dilated tube; last, by assuming that the polymer does not relax by reptation. By comparing the different results, it seems clear that in this case, the reptation process does not occur in the dilated tube. Indeed, because the branches of the pompom molecule represent $80 \%$ of the polymer, reptation in a dilated tube (see eq 20 , with $\Phi_{\text {active }^{2}}=0.04$ ) will always be predicted immediately after the branches fluctuations, whichever the set of parameters. However, in the real case we distinctly see a "shoulder" at low frequencies, coming from the unrelaxed part of the backbone. This represents a failure of the dynamic dilution concept, where time scale separation is not observed, which is corrected in the model by the extended criteria of Graessley. ${ }^{27,28,31,32}$ Furthermore, by comparing the prediction with and without the reptation process, we observe that the results are nearly similar: reptation does not have a strong influence on this pompom polymer relaxation. Similar
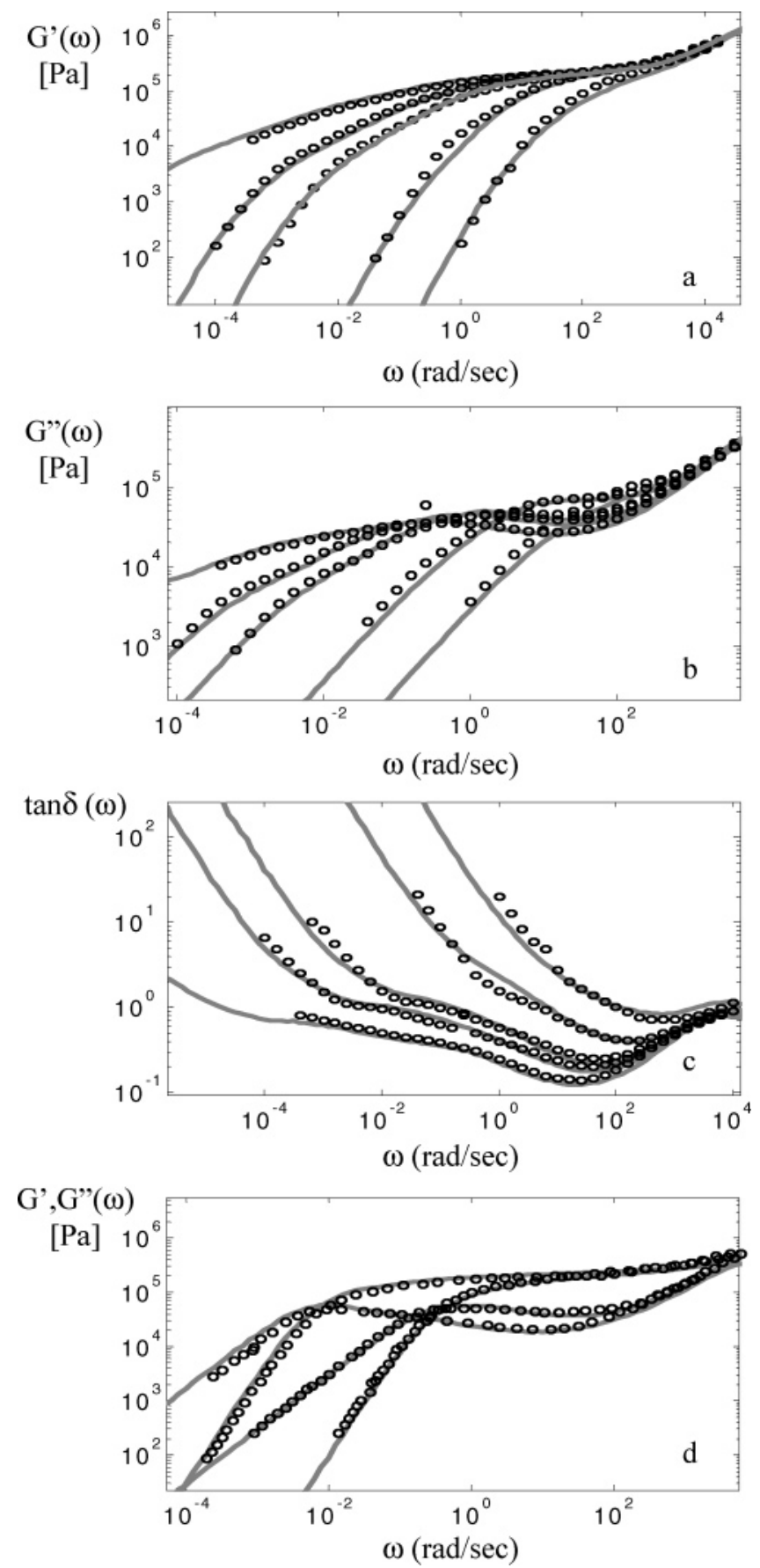

Figure 7. $(\mathrm{a}-\mathrm{c})$ Linear viscoelastic moduli and loss angle $\tan \delta$ of H-samples PS1 to PS5 (see Table 1). Comparison between theoretical (- - -) and experimental (symbols) data. (d) Experimental and predicted moduli for samples PS6 to PS7 (see Table 1).

results were found for the other H-polymer samples, confirming these conclusions. This is a consequence of the large volume fraction of the branches and similar $M_{\mathrm{branch}}$ and $M_{\mathrm{b}}$.

On the other hand, for some of the pompom samples PB1 to PB4, reptation plays a more important role, as shown in Figure 12 (both the shape of $G^{\prime}$ and $G^{\prime \prime}$ and the model predictions). Note that the low-frequency shoulder of $G^{\prime}$ in PB1 (Figure 12a) is probably an experimental issue (maybe not so well-characterized polymer) and does not relate to the backbone reptation. These results indicate that, for reptation to dominate the terminal response, the backbone must be much longer than the branches of these macromolecules.

III.3. Effects of the Polydispersity. Even if the polydispersity considered here is very small $\left(M_{\mathrm{w}} / M_{\mathrm{n}}=1.05\right)$, its effect can be 


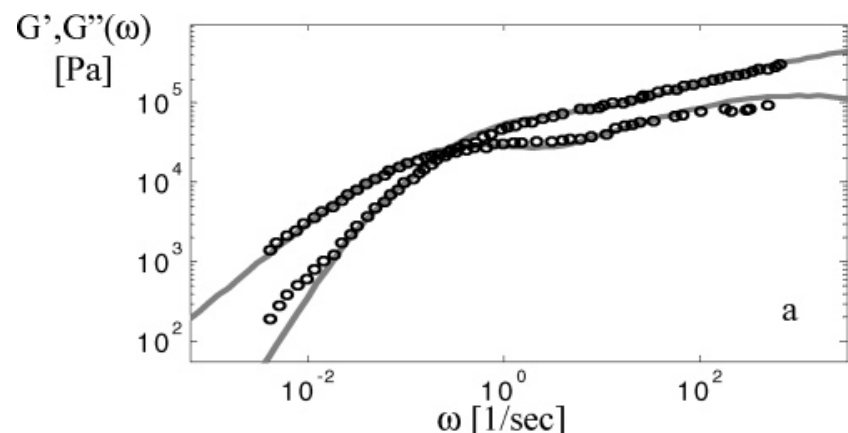

G',G' $(\omega)$
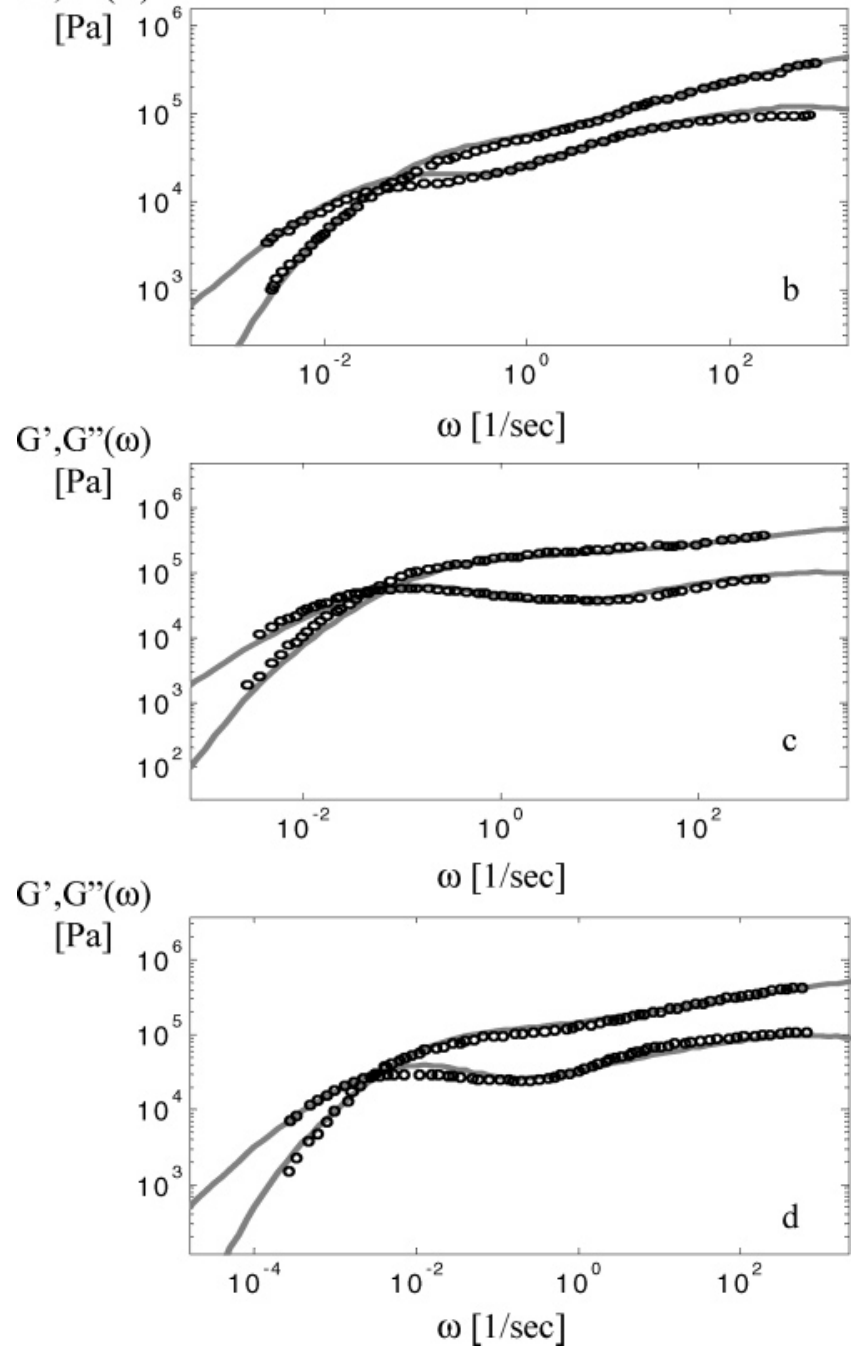

Figure 8. Linear viscoelastic moduli of pompom samples PB1 to PB4 (see Table 1). Comparison between predictions (- - ) and experimental (symbols) data.

very important. ${ }^{17,18}$ This is demonstrated in Figure 13a, which shows the experimental data for the polybutadiene pompom sample PB4, along with the model predictions without and with a polydispersity of 1.05 . This enables us to conclude that these samples are on the average slightly polydisperse. Likewise, Figure $13 \mathrm{~b}$ shows the case of a H-polystyrene (sample PS3). In the latter case, because of the small fraction of backbone (20\%) in comparison to the branches, polydispersity does not affect the predictions appreciably.

III.4. Comparison with the Existing Tube-Model Theories: The Effect of the $p^{2}$ Parameter. In the tube-model theories, originated by Milner and McLeish ${ }^{20}$ and advanced by several groups. ${ }^{14-19,24-26,36,43}$ the parameter $p^{2}$ appears in the equaton of the curvilinear diffusion constant, expressing the
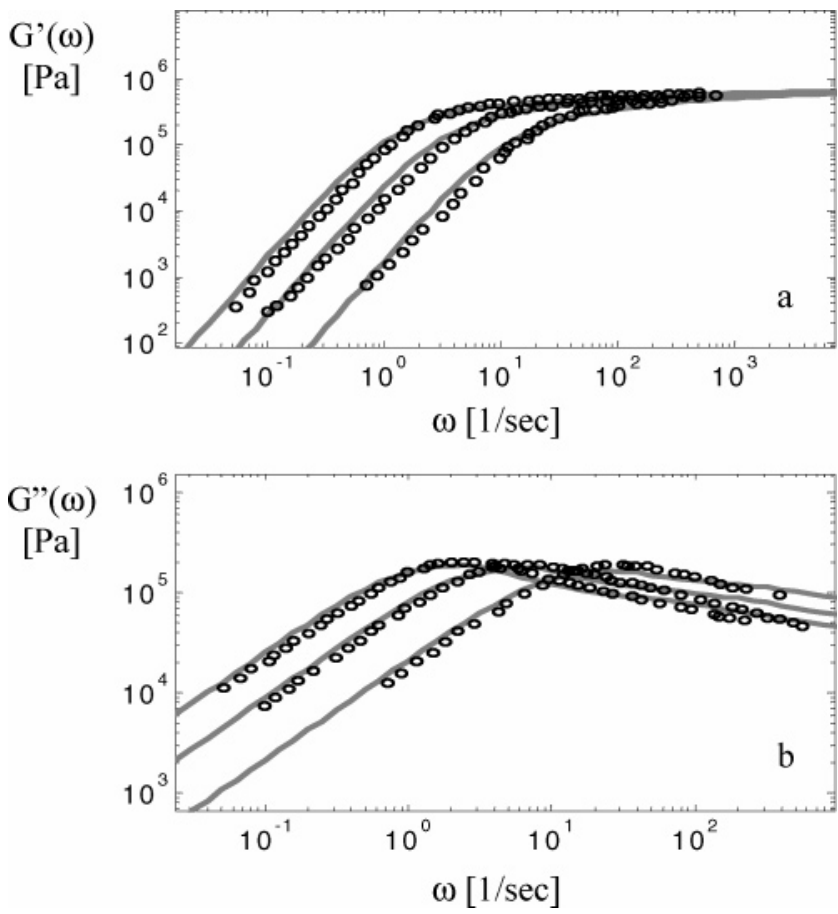

Figure 9. Linear viscoelastic moduli of samples PB5 to PB7 (see Table 1). Comparison between predictions (- - ) and experimental (symbols) data.

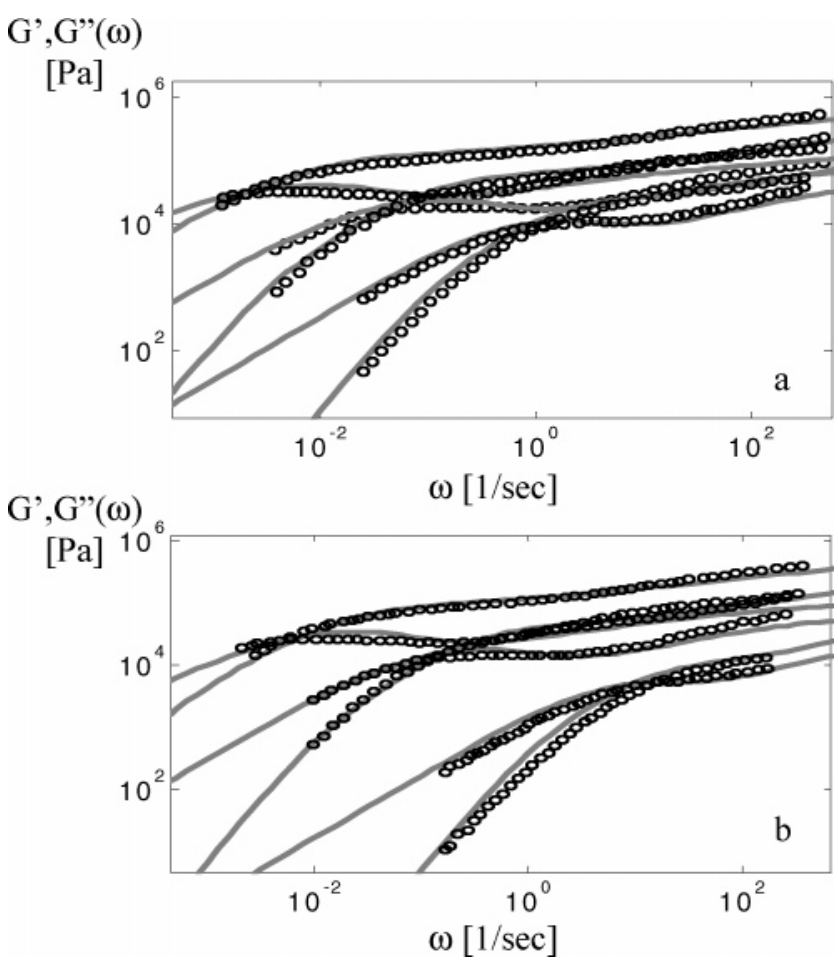

Figure 10. Linear viscoelastic moduli of samples PB8 (see Table 1) diluted in solvent at different concentrations: $\Phi_{\text {polym }}=1,0.73$, and 0.5 (a); $\Phi_{\text {polym }}=0.9,0.63$, and 0.34 (b). Comparison between predictions (- - ) and experimental (symbols) data.

fraction of the tube diameter the branch point moves in a time scale of order $\tau_{\text {branch }}(1)$ :

$$
D_{\mathrm{a}}=\frac{p^{2} a^{2}}{2 \tau_{\text {branch }}(1)}
$$

As explained in the Introduction, $p^{2}$ is usually treated as an adjustable parameter, and assumes a wide range of values. A 

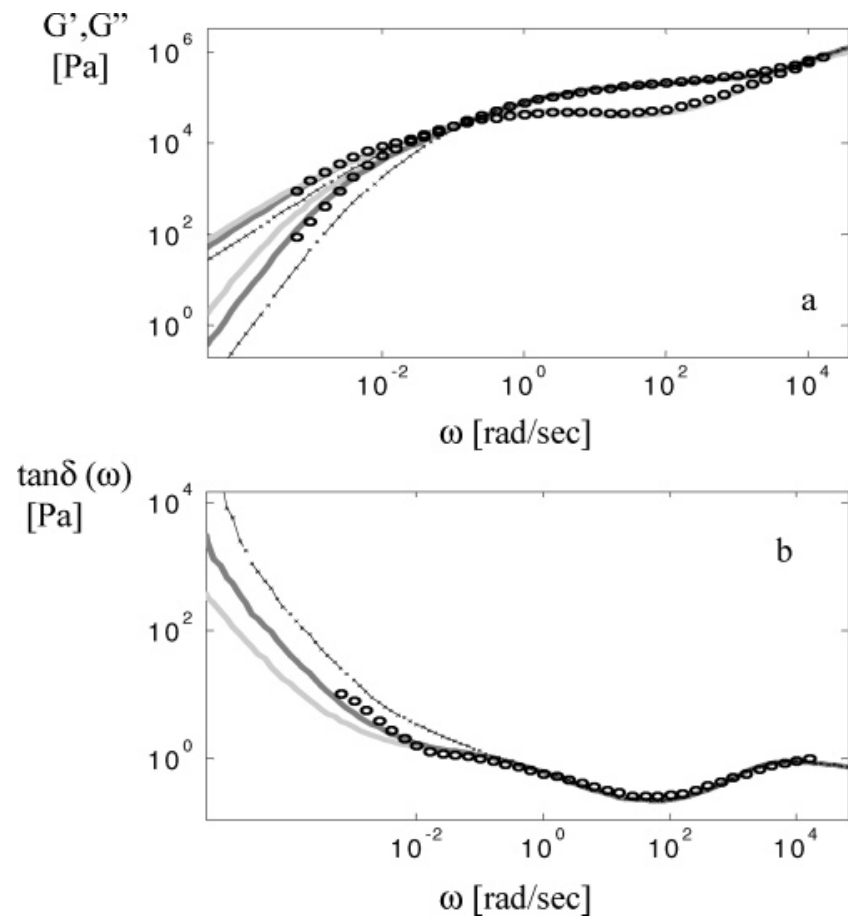

Figure 11. Storage and loss moduli (a) and $\tan \delta$ (b) of sample PS3 calculated without reptation (light gray), by considering the "solvent" polymer fraction described by eqs 19 and 20 (dark gray) or by considering the unrelaxed fraction of the sample equal to the volumetric fraction of the (inner) backbone (black).

value $p^{2}<1$, typically used in most branched polymer studies, implies a slowing- down of the backbone relaxation. ${ }^{14-19,24-26,36,43}$ There are also situations where a value of 1 is used. ${ }^{14,24,25}$ In general, this parameter is considered to depend on chemistry only, ${ }^{14-19}$ although there are suggestions of possible dependence on the macromolecular architecture as well. ${ }^{24-26}$ In the present work, we fix $p^{2}=1$ and there is no need to vary the value of $p^{2}$ across the different samples. This represents a distinct difference that needs being elaborated. Thus, we briefly discuss below the differences in the calculations of the different contributing relaxation processes.

The reptation time of the backbone is given by eqs 19 and 20. Considering that reptation takes place in a (nearly) undiluted tube, this already slows down the backbone relaxation process. However, as shown in the literature, this modification is not enough. ${ }^{24,27-30}$ The main contribution to the slower backbone relaxation, and key difference between the current approach and the previous ones ${ }^{14,15,17,18,24,25,36-43}$ originates from the factor $\left(1-x_{\mathrm{d}}\right)$, which is used in the equation of the reptation time; $x_{\mathrm{d}}$ represents the deeper inner backbone segments relaxed by fluctuations before the reptation process. As already explained in section II.4, to analyze the backbone relaxation by reptation, we consider that the backbone centerof-mass diffuses along its total average equilibrium length. Indeed, even if some segments are already relaxed by fluctuations, they still have a nonzero equilibrium length that we consider here. In fact, the same conclusion was already drawn from the original time-marching model applied to highly asymmetric stars. ${ }^{27}$ Last, the respective difference observed in the calculation of the backbone fluctuations times, which precedes and slows down the backbone reptation process arises principally from the rescaling of the variable $x_{b}$, from the outer free end of a branch to the middle of the molecule (see sections II.1 and II.3).

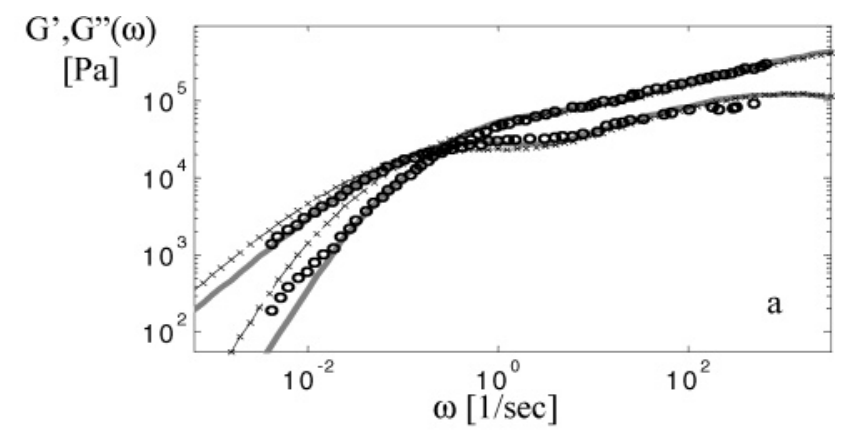

G',G', $(\omega)$

[Pa]
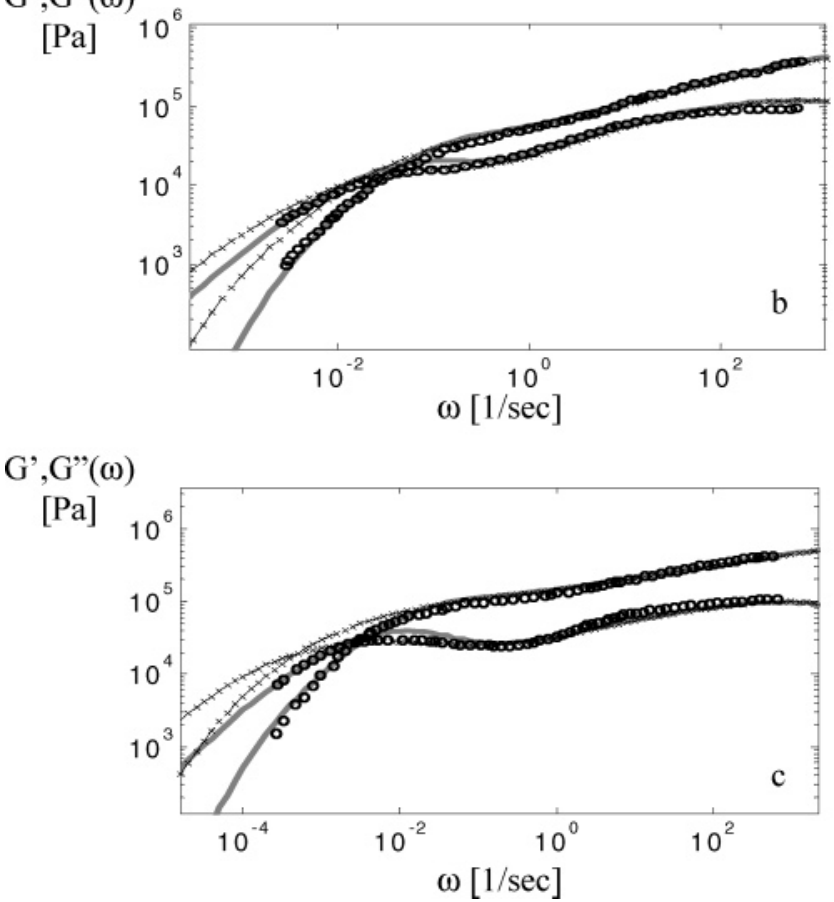

Figure 12. Storage and loss moduli of samples PB1 (a), PB2 (b), and PB4 (c), calculated without any reptation $(\times \times \times)$, or with reptation, by considering that the "solvent" polymer fraction for the backbone is described by eqs 19 and 20 (- - ); see text for details.

\section{Conclusions}

We have developed a general coarse-grained model for predicting the linear viscoelasic properties of branched polymers from the knowledge of their molecular structure and three viscoelastic parameters, i.e., the Rouse time of an entanglement segment, the plateau modulus and the entanglement molecular weight. This is an extension of earlier work using a timemarching algorithm to successfully predict the rheology of linear and star polymers. uses the physics of tube-based theories, originated by McLeish and co-workers. We have appropriately modified and extended this earlier model by proposing a new macromolecular coordinate system and accounting for the diffusion of the branching points and the polydispersity, within the framework of dynamic tube dilation (using the citeria of Graessley). We have obtained excellent predictions for the linear viscoelastic properties of different complex macromolecular architectures, such as $\mathrm{H}$ polymers and pompom polymers, without any adjustable parameters. It has been demonstrated that polydispersity is very important, even when it amounts to $5 \%$ only. There are still some issues at stake, such as the value of the dilution exponent, the validity of the rubber elasticity relation within $15 \%$, and the coupling of the different relaxation modes. Nevertheless, the successful and self-consistent application of the model to different chemistries and architectures gives us confidence that the proposed methodology represents a 

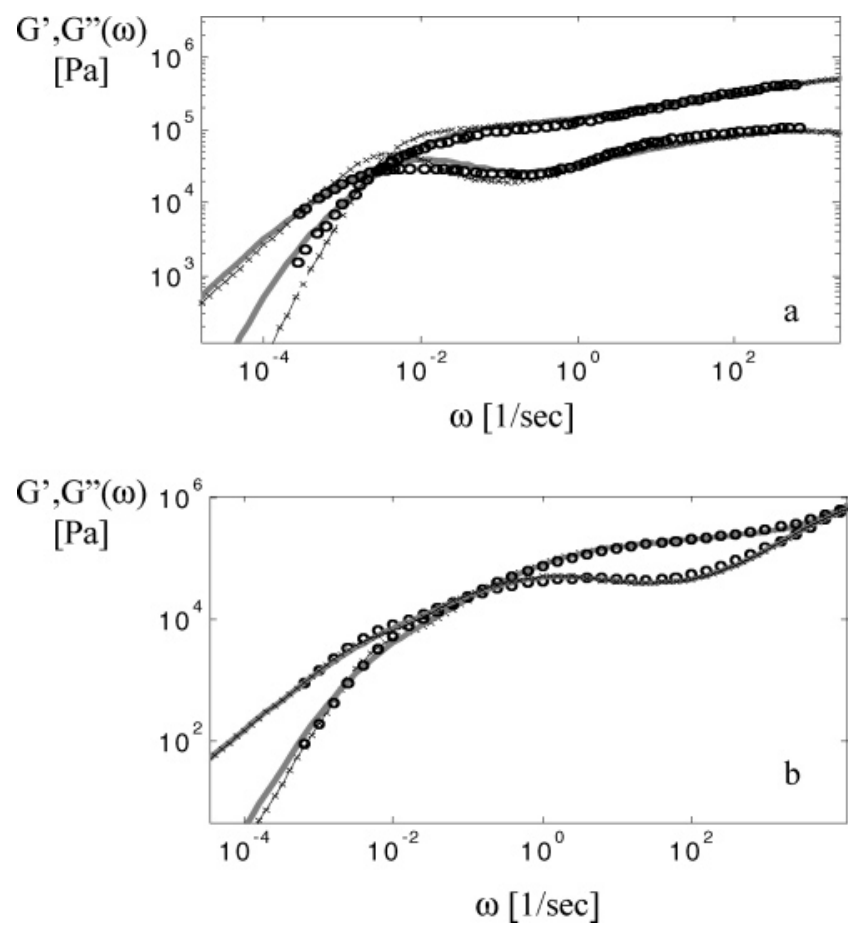

Figure 13. Storage and loss moduli of samples PB4 (a) and PS3 (b), calculated by considering a polydispersity of $1.05(--)$, or by considering the samples as monodisperse $(\times \times \times)$.

powerful generic approach for predicting the linear rheology of branched polymers.

Acknowledgment. Helpful discussions with M. Kapnistos are gratefully acknowledged. This work has been supported by the EU (NoE-Softcomp, Grant NMP3-CT-2004-502235; Individual Marie Curie fellowship- DYCOSYS to E.v.R., Grant MEF-CT-2005-024298).

\section{Appendix I: Relaxation Modulus of a Pompom Molecule}

The relaxation modulus of a monodisperse polymer melt or a concentrated solution is described in general by three terms: the first represents the relaxation of the polymer from its equilibrium state (by reptation, fluctuations, or constraint release), the second is longitudinal modes relaxation, and the third is fast Rouse motion inside the tube: ${ }^{4,7,44}$

$$
\begin{aligned}
& G(t)= \\
& G_{N}{ }^{0} F(t)+\frac{1}{4} G_{N}{ }^{0} F_{\text {Rouse,longitudinal }}(t)+\frac{5}{4} G_{N}{ }^{0} F_{\text {fast Rouse }}(t)
\end{aligned}
$$

The relaxation modulus $F(t)$ of the polymer is calculated by summing up all contributions from segments along the branches and the backbone:

$$
\begin{aligned}
F(t)=\varphi_{\mathrm{b}} & \int_{0}^{1}\left(p_{\text {rept }}\left(x_{\mathrm{b}}, t\right) p_{\text {fluc }}\left(x_{\mathrm{b}}, t\right) p_{\text {envir }}\left(x_{\mathrm{b}}, t\right)\right) \mathrm{d} x_{\mathrm{b}}+ \\
& \varphi_{\text {branch }} \int_{0}^{1}\left(p_{\text {fluc }}\left(x_{\text {branch }}, t\right) p_{\text {envir }}\left(x_{\text {branch }}, t\right)\right) \mathrm{d} x_{\text {branch }}
\end{aligned}
$$

A given segment will contribute to the modulus if it has not been relaxed by any of the three possible relaxation mechanisms: $p_{\text {rept }}, p_{\text {fluc }}$, and $p_{\text {envir }}$ represent the probabilities that the segment $x$ remains oriented by reptation, fluctuations or constraint release, respectively. These terms are described in sections II.3, II.4 and II.5. Because the reptation and the fluctuations times of a segment are not necessary timeindependent (for example they may decrease with increasing the polymer fraction acting as a solvent), we use a timemarching algorithm, ${ }^{27}$ which determines the survival probabilities of each segment at a given time $t_{k}$ from the corresponding values at the previous time $t_{k-1}$ :

$$
\begin{aligned}
& p_{\text {fluc }}\left(x_{i}, t_{k}\right)=p_{\text {fluc }}\left(x_{i}, t_{k-1}\right) p_{\text {fluc }}\left(x_{i},\left[t_{k-1}, t_{k}\right]\right) \\
& p_{\text {rept }}\left(x_{i}, t_{k}\right)=p_{\text {rept }}\left(x_{i}, t_{k-1}\right) p_{\text {rept }}\left(x_{i},\left[t_{k-1}, t_{k}\right]\right)
\end{aligned}
$$

The survival probability at $t_{k}$ is the product of the survival probability at $t_{k-1}$ and the survival probability during the time interval taken between $t_{k-1}$ and $t_{k}$. The latter probabilities are calculated from the values of the relaxation times $\tau_{\text {relax }}\left(x_{i}, t_{k}\right)$ calculated at time $t_{k}$, after the update of $\Phi\left(t_{k}\right)$, the polymer fraction acting as a solvent in the reptation and fluctuation processes:

$$
p_{\text {survival }}\left(x_{i},\left[t_{k-1}, t_{k}\right]\right)=\exp \left(\frac{-(\Delta t)}{\tau_{\text {relax }}\left(x_{i}, t_{k}, \Phi\left(t_{k}\right)\right)}\right)
$$

The time $\tau_{\text {relax }}$ represents either the reptation time or the fluctuations time of the segment $x_{i}$. The second and third terms, calculated as in ref 7, are functions of the Rouse time of the backbone and of the branches

$$
\begin{gathered}
F_{\text {Rouse,longitudinal }}=\left(\frac{1}{Z} \varphi_{\mathrm{b}} \sum_{j=1}^{Z-1} \exp \left(-\frac{j^{2} t}{\tau_{\text {Rouse }}\left(M_{\mathrm{b}}+2 M_{\text {branch }}\right)}\right)+\right. \\
\left.\frac{1}{Z} \varphi_{\text {branch }} \sum_{j=1}^{Z-1} \exp \left(-\frac{j^{2} t}{\tau_{\text {Rouse }}\left(M_{\text {branch }}\right)}\right)\right)(\mathrm{I}-6) \\
F_{\text {fast Rouse }}=\left(\frac{1}{Z} \varphi_{\mathrm{b}} \sum_{j=Z}^{N} \exp \left(-\frac{2 j^{2} t}{\tau_{\text {Rouse }}\left(M_{\mathrm{b}}+2 M_{\text {branch }}\right)}\right)+\right. \\
\left.\frac{1}{Z} \varphi_{\text {branch }} \sum_{j=Z}^{N} \exp \left(-\frac{2 j^{2} t}{\tau_{\text {Rouse }}\left(M_{\text {branch }}\right)}\right)\right)
\end{gathered}
$$

where $Z$ is the number of entanglements and $N$, the number of Khun segments, and

$$
\tau_{\text {Rouse }, M_{i}}=\tau_{\mathrm{e}}\left(\frac{M_{i}}{M_{\mathrm{e}}}\right)^{2}, \quad i=\text { branch, } b
$$

$M_{e}$ is the entanglement molecular weight and $\tau_{e}$ is its Rouse time of an entanglement segment:

$$
\tau_{\mathrm{e}}=\frac{\zeta_{0} b^{2}}{3 \pi^{2} k T}\left(\frac{M_{\mathrm{e}}}{m_{0}}\right)^{2}
$$

where $\zeta_{0}$ represents the monomeric friction coefficient. The storage and loss moduli are then calculated from the relaxation modulus $G(t)$ by using the Schwarzl relations, which are an approximation of the Fourier transform. 6,45

\section{Appendix II: Branches Fluctuations}

The branches of a pompom molecule relax exactly as the arms of a star molecule. As described by Milner and McLeish, ${ }^{20}$ each branch has an equilibrium length, $L_{\mathrm{eq}}\left(M_{\text {branch }}\right)$, coming from the balance between an entropic force which tends to reduce the distance between the end chain and the branching point, and a topological force, representing the effect of the environment, which prevents this retraction. Because the real length of the branch fluctuates about this equilibrium length (which is the most probable), the initial tube segments relax little by little, from the outer to the inner one. Their corresponding fluctuations 
time increases exponentially with the depth of the segment:

$$
\begin{gathered}
U\left(x_{\text {branch }}\right)=\frac{3 k T}{2 N b^{2}}\left(L_{\text {eq }} x_{\text {branch }}\right)^{2}+\text { const } \\
\ln \tau_{\text {late }}\left(x_{\text {branch }}\right)=\frac{U\left(x_{\text {branch }}\right)}{k T}+\text { const }
\end{gathered}
$$

Since the fluctuations times of the segments are exponentially dependent on their location on the branch, we consider that the external segments already relaxed act as a solvent for the relaxation of the inner segments. This is the idea of dynamic dilution: ${ }^{22,23}$ if a fraction $\left(1-\Phi\left(t_{k}, x_{\text {branch }}\right)\right)$ of the polymer acts as a solvent, the molecular weight between two effective entanglements (which have an effective role in the orientation of the polymer $), M_{\mathrm{e}}\left(t_{k}, x_{\text {branch }}\right)$, increases

$$
M_{\mathrm{e}}\left(t, x_{\text {branch }}\right)=\frac{M_{\mathrm{e}, 0}}{\Phi\left(t_{k}, x_{\text {branch }}\right)^{\alpha}}
$$

where $M_{\mathrm{e}, 0}$ is the nominal entanglement molecular weight (before dilution) and the parameter $\alpha$ is the dilution exponent, usually between 1 and 4/3. ${ }^{42,46}$ This effect must be included in the expression II-2:

$$
\frac{\partial \ln \tau_{\text {late }}\left(x_{\text {branch }}\right)}{\partial x_{\text {branch }}}=3\left(\frac{M_{\text {branch }}}{M_{\mathrm{e}}\left(t_{k}, x_{\text {branch }}\right)}\right) x_{\text {branch }}
$$

The function $\Phi\left(t_{k}, x_{\text {branch }}\right)$ represents the unrelaxed part of the pompom polymer, when the branches are relaxed until the segment defined by the normalized variable $x_{\text {branch }}$, at time $t_{k}$. In this particular case, because the reptation process occurs only after the relaxation of the branches has been completed, no "dynamic reptation solvent" is taken into account in $\Phi\left(t_{k}, x_{\text {branch }}\right)$. Therefore, this variable does not depend on the time:

$\Phi\left(t_{k}, x_{\text {branch }}\right)=\Phi\left(x_{\text {branch }}\right)=\varphi_{\mathrm{b}}\left(1-x_{\mathrm{b}}\right)+\varphi_{\text {branch }}\left(1-x_{\text {branch }}\right)$

In this expression, the value of $x_{\mathrm{b}}$, which is defined as in Figure 1 , is directly calculated from $x_{\text {branch: }}$

$$
x_{\mathrm{b}}=\frac{x_{\text {branch }} M_{\mathrm{branch}}}{M_{\mathrm{branch}}+M_{\mathrm{b}} / 2}
$$

As introduced in ref 20, for segments having a potential $U\left(x_{\mathrm{a}}\right)$ $<k T$, their early fluctuations times do not follow eq II.4 but are described by a Rouse process:

$$
\tau_{\text {early }}\left(x_{\text {branch }}\right)=\frac{9 \pi^{3}}{16}\left(\frac{M_{\text {branch }}}{M_{\mathrm{e}, 0}}\right)^{2} \tau_{\mathrm{R}, \text { branch }} x_{\text {branch }} 4
$$

where $\tau_{\mathrm{R} \text {,branch }}$ is the Rouse time of the branch. No polymer solvent is considered in this equation. Indeed, to act as a solvent, the relaxed part of the polymer must be well-separated in time scale from the relaxing segments. This is not observed here because of the speed of the Rouse relaxation.

The transition between the two fluctuation processes happens at a transition segment $x_{\text {trans }}$, for which the potential $U\left(x_{\text {trans }}\right)=$ $k T$, and it is described as

$$
\begin{array}{r}
\tau_{\text {fluc }}\left(x_{\text {branch }}\right)=\tau_{\text {early }}\left(x_{\text {branch }}\right) \quad \text { for } x_{\text {branch }}<x_{\text {trans }} \\
\tau_{\text {fluc }}\left(x_{\text {branch }}\right)=\tau_{\text {early }}\left(x_{\text {trans }}\right) \exp \left(\frac{\Delta U\left(x_{\text {trans }} \rightarrow x_{\text {branch }}\right)}{k T}\right) \\
\text { for } x_{\text {branch }}>x_{\text {trans }}
\end{array}
$$

By using these expressions, we do not need to calculate any waiting time $\tau_{0}$, contrary to the other approaches in the literature..$^{20}$

\section{References and Notes}

(1) de Gennes, P. G. J. Chem. Phys. 1971, 55, 527.

(2) Doi, M.; Edwards, S. F. The Theory of Polymer Dynamics; Oxford University Press: New York, 1986.

(3) Watanabe, H. Prog. Polym. Sci. 1999, 24, 1253.

(4) Marrucci, G.; Greco, F.; Ianniruberto, G. Curr. Opin. Colloid Interface Sci. 1999, 4, 283. Evaraers, R.; Sukumaran, S. K.; Grest, G. S.; Svaneborg, C.; Sivasubramanian, A.; Kremer, K. Science 2004, 303, 823. Likhtman, A. E. Macromolecules 2005, 38, 6128.

(5) McLeish, T. C. B. Adv. Phys. 2002, 51, 1379.

(6) van Ruymbeke, E.; Keunings, R.; Stephenne, V.; Hagenaars, A.; Bailly, C. Macromolecules 2002, 35, 2689.

(7) Likhtman, A. E.; McLeish, T. Macromolecules 2002, 35, 6332.

(8) Hsieh, H. L.; Quirk, R. P. Anionic Polymerization: Principles and Practical Applications; Marcel Dekker: New York, 1996.

(9) Pitsikalis, M.; Pispas, S.; Mays, J. W.; Hadjichristidis, N. Adv. Polym. Sci. 1998, 135, 1.

(10) Hadjichristidis, N.; Pitsikalis, M.; Pispas, S.; Iatrou, H.; Vlahos, C. Adv. Polym. Sci. 1999, 142, 71 .

(11) Roovers, J.; Graessley, W. W. Macromolecules 1981, 14, 766. Roovers, J. Polymer 1979, 20, 843. Roovers, J.; Toporowski, P. Macromolecules 1987, 20, 2300.

(12) Knauss, D. M.; Al-Muallem, H. A.; Huang, T.; Wu, D. T. Macromolecules 2000, 33, 3557.

(13) Larson, R. G. Macromolecules 2001, 34, 4556.

(14) McLeish, T. C. B.; Allgaier, J.; Bick, D. K.; Bishko, G.; Biswas, P.; Blackwell, R.; Blottiere, B.; Clarke, N.; Gibbs, B.; Groves, D. J.; Hakiki, A.; Heenan, R. K.; Johnson, J. M.; Kant, R.; Read, D. J. Young, R. N. Macromolecules 1999, 32, 6734

(15) Daniels, D. R.; McLeish, T. C. B.; Crosby, B. J.; Young, R. N.; Fernyhough, C. M. Macromolecules 2001, 34, 7025.

(16) Blackwell, R. J.; Harlen, O. G.; McLeish, T. C. B. Macromolecules 2001, 34, 2579.

(17) Kapnistos, M.; Vlassopoulos, D.; Roovers, J.; Leal, L. G. Macromolecules 2005, 38, 7852.

(18) Kapnistos, M.; Koutalas, G.; Hadjichristidis, N.; Roovers, J.; Lohse, D. J.; Vlassopoulos, D. Rheol. Acta, submitted, 2006.

(19) McLeish, T. C. B.; Larson, R. G. J. Rheol. 1998, 42, 81.

(20) Milner, S. T.; McLeish, T. C. B. Macromolecules 1997, 30, 2159.

(21) Milner, S. T.; McLeish, T. C. B. Macromolecules 1998, 31, 7479

(22) Marrucci, G. J. Polym. Sci., Polym. Phys. Ed. 1985, 23, 159.

(23) Ball, R. C.; McLeish, T. C. B. Macromolecules 1989, 22, 1911. McLeish, T. C. B. J. Rheol. 2003, 47, 177.

(24) Frischknecht, A. L.; Milner, S. T.; Pryke, A.; Young, R. N.; Hawkins, R.; McLeish, T. C. B. Macromolecules 2002, 35, 4801.

(25) Lee, J. H.; Fetters, L. J.; Archer, L. A. Macromolecules 2005, 38, 4484.

(26) Lee, J. H.; Fetters, L. J.; Archer, L. A. Macromolecules 2005, 38, 10763.

(27) van Ruymbeke, E.; Keunings, R.; Bailly, C. J. Non-Newtonian Fluid Mech. 2005, 128, 7.

(28) Struglinski, M. J.; Graessley, W. W. Macromolecules 1985, 18, 2630.

(29) Tsenoglou, C. ACS Polym. Prepr. 1987, 28, 185. Tsenoglou, C. Macromolecules 1991, 24, 1762.

(30) des Cloizeaux, J. J. Europhys. Lett. 1988, 5, 437.

(31) Watanabe, H.; Ishida, S.; Matsumiya, Y.; Inoue, T. Macromolecules 2004, 37, 1937.

(32) Watanabe, H.; Ishida, S.; Matsumiya, Y.; Inoue, T. Macromolecules 2004, 37, 6619. Watanabe, H.; Matsumiya, Y.; Inoue, T. Macromolecules 2002, 35, 2339.

(33) Park, S. J.; Larson, R. G. Macromolecules 2004, 37, 597.

(34) Roovers, J. Macromolecules 1984, 17, 1196.

(35) Archer, L. A.; Varshney, S. K. Macromolecules 1998, 31, 6348

(36) Juliani; Archer, L. A. Macromolecules 2002, 35, 10048. Archer, L. A.; Juliani Macromolecules 2004, 37, 1076.

(37) Wesslau, H. Makrom Chemie 1956, 20, 111.

(38) Graessley, W. W.; Roovers, J. Macromolecules 1979, 12, 959. 
(39) Larson, R. G.; Sridhar, T.; Leal, L. G.; McKinley, G. H.; Likhtman, A. E.; McLeish, T. C. B. J. Rheol. 2003, 47, 809. Fetters, L. J.; Lohse, D. J.; Richter, D.; Witten, T. A.; Zirkel, A. Macromolecules 1994, 27, 4639 .

(40) Ferry, J. D. Viscoelastic Properties of Polymers, 3rd ed.; Wiley: New York, 1980.

(41) Liu, C. Y.; He, J.; Keunings, R.; Bailly, C. Do tube models yield consistent predictions for the relaxation time and apparent plateau modulus of entangled linear polymers? Macromolecules 2006, in press.

(42) Rubinstein, M.; Colby, R. H. Polymer Physics; Oxford University Press: New York, 2003. Colby, R. H.; Rubinstein, M. Macromolecules 1990, 23, 2753.
(43) Frischknecht, A. L.; Milner, S. T. Macromolecules 2000, 33, 9764.

(44) Miros, A.; Vlassopoulos, D.; Likthman, A. E.; Roovers, J. J. Rheol. 2003, 47, 163 .

(45) Schwarzl, F. R. Rheol. Acta 1971, 10, 166

(46) Raju, V. R.; Menezes, E. V.; Marin. G.; Graessley, W. W. Macromolecules 1981, 14, 1668. Daniels, D. R.; McLeish, T. C. B.; Kant, R.; Crosby, B. J.; Young, R. N.; Pryke, A.; Allgaier, J.; Groves, D. J.; Hawkins, R. J. Rheol. Acta 2001, 40, 403. Tao, H.; Huang, C.; Lodge, T. P. Macromolecules 1999, 32, 1212. Park, S. J.; Larson, R. G. J. Rheol. 2003, 47, 199 .

MA0604385 